\title{
BCMA CAR T-cell therapy arrives for multiple myeloma: a reality
}

\author{
Shih-Feng Cho ${ }^{1,2,3}$, Kenneth C. Anderson ${ }^{1}$, Yu-Tzu Tai ${ }^{1}$ \\ ${ }^{1}$ LeBow Institute for Myeloma Therapeutics and Jerome Lipper Multiple Myeloma Center, Dana-Farber Cancer Institute, Harvard Medical School, \\ Boston, MA, USA; ${ }^{2}$ Division of Hematology \& Oncology, Department of Internal Medicine, Kaohsiung Medical University Hospital, Kaohsiung \\ Medical University, Kaohsiung, Taiwan, China; ${ }^{3}$ Faculty of Medicine, College of Medicine, Kaohsiung Medical University, Taiwan, China \\ Correspondence to: Yu-Tzu Tai, PhD. Department of Medical Oncology, Dana-Farber Cancer Institute, M551, 450 Brookline Avenue, Boston, MA \\ 02215, USA. Email: yu-tzu_tai@dfci.harvard.edu. \\ Provenance: This is an invited Editorial commissioned by Section Editor Liang Wang, MD (Zhujiang Hospital of Southern Medical University, \\ Guangzhou, China). \\ Comment on: Brudno JN, Maric I, Hartman SD, et al. T Cells Genetically Modified to Express an Anti-B-Cell Maturation Antigen Chimeric Antigen \\ Receptor Cause Remissions of Poor-Prognosis Relapsed Multiple Myeloma. J Clin Oncol 2018;36:2267-80.
}

Submitted Oct 22, 2018. Accepted for publication Nov 06, 2018.

doi: $10.21037 / \mathrm{atm} .2018 .11 .14$

View this article at: http://dx.doi.org/10.21037/atm.2018.11.14

\section{Introduction}

In the recent years, the development of immunotherapy has revolutionized the cancer treatment including multiple myeloma (MM). Therapeutic agents killing MM cells and activating immune cell function to overcome immunocompromised microenvironment further improved clinical outcome. Importantly, adoptive cellular therapy using chimeric antigen receptor (CAR) $\mathrm{T}$ cell targeting specifically expressed antigen on tumor cells has become one of the exciting breakthrough in MM immunotherapy.

\section{The Brudno et al. study}

The complete report of the first-in-human anti-B-cell maturation antigen (BCMA) CAR T cell therapy clinical trial was just published in August 2018 following earlier results reported by Ali et al. in 2016 (1). In this phase 1 study, 24 patients were enrolled with heavily treated (range of prior lines of treatment: 3-19, median: 9.5) relapsed and refractory MM. The patients received CAR-T therapy with doses ranging from $0.3 \times 10^{6}$ to $9 \times 10^{6} / \mathrm{kg}$ after administration of chemotherapy with cyclophosphamide (300 mg/m $\mathrm{m}^{2}, 3$ days) and fludarabine $\left(30 \mathrm{mg} / \mathrm{m}^{2}, 3\right.$ days). A total of 16 patients received highest dose level of CAR T cell infusion. Among these patients, the overall response rate was $81 \%$, including 2 stringent complete response, 8 very good partial response, and 3 partial response. All 11 evaluated patients achieved negativity for minimal residual disease by flow cytometry. The median event-free survival for patients received highest dose level was 31 weeks. During follow-up, six patients treated with highest dose level of CAR T cell infusion have continuous responses. The other ten patients experienced progression of MM. With respect to immunomodulatory effect, CD8+ CAR T cells proliferated and differentiated. The CD4/CD8 ratio decreased significantly after infusion. Moreover, infused CAR $T$ cells showed more differentiated phenotype like effector memory and effector memory $\mathrm{T}$ cells. Regarding toxicity profile, cytokine-releasing syndrome (CRS) was obvious if the percentage of MM cells in bone marrow was high at the time of CAR T cell infusion. Besides, grade 3/4 hematologic adverse including leukopenia or neutropenia was observed in 15 patients $(15 / 16,93.8 \%)$ received highest dose of CAR T cell infusion. No mortality was noted in this study.

This study confirmed that CAR T cell therapy is an effective therapeutic intervention even in RRMM patients. In addition, this study provided valuable information for further clinical studies and practice on anti-MM CAR-T cell treatment. First, increased number of CAR T cell was found in responders, which reached a peak between 1 and 2 weeks after infusion. High peak CAR T cell number was linked to better responses, concordant with the finding in anti-CD19 CAR T clinical trial in acute lymphoblastic leukemia (ALL). Second, the toxicity of CAR T therapy was substantial in this study. For example, some patients with 
severe CRS need vasopressor treatment. Like the study of anti-CD19 CAR T in ALL, this study also showed that high tumor burden (high percentage of plasma cells in bone marrow) was also associated with a higher risk of severe CRS. Third, decrease or even loss of BCMA expression on MM cells was observed after anti-BCMA CAR T cell infusion. The serum BCMA level significantly decreased in responders compared with non-responders. However, some BCMA-negative MM cells were detected when the disease progressed. This finding suggests that antigen escape and downregulation might emerge as key issues impacting the durability of CAR T cell therapy.

\section{Anti-BCMA CAR01 $T$ cell therapy in MM immunotherapy}

BCMA, a member of tumor necrosis factor, plays a vital role in supporting differentiation and survival of plasma cells. It is selectively expressed on normal plasma cells and MM cells $(2,3)$. In contrast, cells of other tissues have undetectable expression of BCMA. Together with transmembrane activator and calcium modulator and cyclophilin ligand interactor (TACI), BCMA is also the receptor for A proliferation-inducing ligand (APRIL). In MM, APRIL/ BCMA pathway plays a key role in supporting growth, drug resistance and immunocompromised environment $(4,5)$. Because of its exclusive expression on plasma cells and significant role in MM, BCMA has emerged as an ideal therapeutic target in MM treatment $(6,7)$.

CAR $\mathrm{T}$ cell therapy for cancer treatment has begun a new era in medicine. CAR is a synthetic molecule composed of an extracellular antigen recognition domain, intracellular signal domains, and costimulatory domains. By engineering $\mathrm{T}$ cells with CAR, CAR T cells identify and bind to antigen on tumor cell independent of HLA. After binding to tumor cells, an immune synapse was formed followed by activation and proliferation of cytotoxic T cells, as well as cytokine production, leading to killing of targeted tumor cells $(8,9)$. The first CAR-T therapy, anti-CD19 CAR T cells was investigated on patients with ALL (10), which showed impressive response rate in heavily pretreated relapsed and refractory patients. In term of safety, the most notable side effect of CAR T cell therapy was CRS which was associated with increased serum level of inflammatory cytokines. With close monitor of vital sign, along with timely and adequate treatment, this complication is manageable $(11,12)$.

Following the successful story of anti-CD19 CAR $\mathrm{T}$ cell therapy on ALL, the first anti-BCMA CAR was synthesized by National Cancer Institute in 2013 (2). The associated preclinical study further confirmed that BCMA was exclusively expressed on malignant and normal plasma cells at mRNA and protein levels. Importantly, anti-BCMA CAR T cells successfully targeted BCMA-expressing MM cells and eradicated them. Simultaneously, multiple BCMAbased treatment modalities were developed including antibody-drug conjugate, bispecific T-cell engager, bispecific molecule, and bi/trispecific antibodies $(3,7)$. Among these novel interventions, CAR-T cell therapy is the first one entering clinical studies (7).

In 2016, the preliminary data regarding safety and efficacy of anti-BCMA CAR T therapy was revealed (13). There were twelve heavily pretreated relapse and refractory MM (RRMM) patients enrolled in this first anti-BCMA CAR T study using a murine single-chain variable fragment (scFv) against human BCMA and CD28 costimulatory domain. These twelve patients receiving different doses of CAR T cells were administered following conditioning chemotherapy (13). In another study reported by Cohen et al. (14), an anti-BCMA CAR with full humanized scFv and $4-1 B B$ costimulatory domain was synthesized. Six heavily pretreated RRMM patients were enrolled and received different doses of CAR $\mathrm{T}$ cell infusion without conditioning chemotherapy (14). Both studies showed acceptable safety profile and promising response rate. These exciting data highlighted the therapeutic potential of CAR T therapy, leading to multiple clinical studies of antiBCMA CAR T-cell therapy in MM.

\section{Perspectives and conclusions}

The Brudno et al. study first demonstrated that CAR T-cell therapy can achieve impressive clinical response in heavily pretreated RRMM patients and further proved that BCMA is a suitable target for immunotherapy. As CAR T-cell therapy is a potent immunotherapy, the targeted antigen must be highly and specifically expressed on the surface of tumor cells to avoid off-target toxicities. BCMA is an ideal target for CAR T-cell therapy since its expression is highly restricted to MM cells and plasma cells, indeed, the toxic effect of anti-BCMA CAR T cells on normal cells is minimal (15). However, application of CAR T-cell therapy in cancer treatment including $M M$ is still facing several challenges, including optimization of persistence and survival of CAR T cells in human body, substantial toxic effects of conditional chemotherapy or CAR T-cell therapy, and antigen escape related disease progression. Besides, 
there is still no data of long-term follow-up to know how long the efficacy of CAR T-cell therapy can last.

To optimize CAR T therapy, several strategies for future CAR development are considered (16). For example, murine single-chain variable fragment $(\mathrm{scFv})$ is more immunogenic and may limit persistence of CAR T cells. Some new CARs which utilize humanized scFv are under evaluation. Besides, the addition of costimulatory domain like CD28, 4-1BB, or both significantly enhance expansion and persistence of CAR T cells, as well as exhibition of more anti-tumor activity. However, the risk of CRS increases obviously because more cytokines are produced. High percentages of CRS suggest that patients' vital sign should be monitor closely. The patients may need to stay in intensive care unit, resulting in another barrier of delivering CAR T-cell therapy in clinical setting. Novel forms of CAR T-cell therapy such as transfection with mRNA coding BCMA CAR to T cells (17) or implantation of a suicidal gene like inducible caspase 9 and truncated epidermal growth factor receptor to regulate persistence of CAR T cells are under development and investigation (18). To make CAR-T therapy more cost-effective and acceptable, novel allogenic donor derived CAR $\mathrm{T}$ cells modified by gene editing technologies to generate off-the-shelf CAR T cells are under development and related clinical studies are ongoing (19).

With respect to CAR T-cell therapy in MM, the early report of this study (reported by Ali et al.) indicated higher dose may be necessary to achieve higher response rate (13). Based on this finding, the majority of following enrolled and evaluated patients in Brudno et al. final report received highest dose of CAR T cell infusion $\left(9 \times 10^{6} / \mathrm{kg}\right)$. AntiBCMA CAR T-cell study (bb2121) also suggested that dose levels above $50 \times 10^{6}$ CAR T cells (total number) can achieve high response rate (20). However, more studies are still needed to define the best effective and safe doses.

The Brudno et al. study also used conditioning chemotherapy before CAR T cells infusion. However, high percentage of grade $3 / 4$ hematologic adverse events were observed. The rationale for conditioning therapy was based on finding of some preclinical studies. For example, cytotoxic function and subsequent persistence of $\mathrm{T}$ cells can be enhanced by lymphodepleting conditioning therapy prior to adoptive $\mathrm{T}$ cell administration (21). Additionally, conditioning can deplete $\mathrm{T}$ regulatory cells, leading to enhancement of CAR T cell therapy (22). In a CLL clinical study, administration of cyclophosphamide conditioning chemotherapy before anti-CD19 CAR T cell infusion can reduce tumor burden and increase persistence of infused T cells (23). Based on these findings, application of conditioning chemotherapy should consider patients' performance. As one-third of newly diagnosed MM patients are older than 75 years and more that $30 \%$ of them are frail, these factors would be barriers to use CAR T-cell therapy or incorporation of conditioning chemotherapy to CAR T-cell therapy (24).

Additional strategies may be applicable to improve clinical outcome in anti-MM CAR T-cell therapy. For example, $M M$ is genetically and phenotypically heterogeneous, newer CAR which targets other MM related antigen like CD38, CD138 or signaling lymphocyte-activating molecule 7 is also under investigation. Combined BCMA CAR with another CAR targeting another antigen may reduce the risk of relapse due to tumor antigen escape. For patients with huge MM burden, using other anti-MM agent to decrease MM cells followed by CAR T cell therapy would be another flexible strategy. In addition, repeated infusions with lower number of CAR T cells can be tried in the clinical trial to answer if this strategy can reduce risk of CRS.

Since CAR T-cell therapy yielded high response in RRMM patients, another question is whether CAR T-cell therapy should move to earlier line therapy. Currently, the development of CAR T-cell therapy in MM is still in early stage, it remains too early to be answered. However, some factors should be considered, including the toxicity of CAR T-cell therapy and the feasibility of delivering the drug. If these two factors can be further improved, then CAR T-cell therapy could be moved up and works well.

In summary, the Brudno et al. study demonstrated anti-BCMA CAR T-cell therapy has promising clinical activity. The most exciting finding of this study is that CAR T-cell therapy kills MM cells highly resistant to previous standard therapies to achieve durable responses in the RRMM patients. Since it may further prolong survival in these sickest MM patients, anti-BCMA CAR $\mathrm{T}$ therapy may be a potentially cost-effective therapeutic intervention. Severe CRS, as the major side effect of CAR T-cell therapy, was observed in some patients, but it was manageable. Furthermore, early but promising results of other anti-BCMA CAR T-cell clinical studies were also reported (20,25). As the field of CAR T-cell therapy in MM immunotherapy is rapidly growing, this study set the stage for researches to do fine-tuning on the right target, dosage, and conditioning chemotherapy, as well as continuously develop safer CAR T-cell therapy for MM. 


\section{Acknowledgements}

Funding: The funding was provided by National Institutes of Health National Cancer Institute Specialized Programs of Research Excellence grants P50100707, R01CA207237, and RO1 CA050947.

\section{Footnote}

Conflicts of Interest: KC Anderson is an American Cancer Society Clinical Research Professor. The other authors have no conflicts of interest to declare.

\section{References}

1. Brudno JN, Maric I, Hartman SD, et al. T Cells Genetically Modified to Express an Anti-B-Cell Maturation Antigen Chimeric Antigen Receptor Cause Remissions of Poor-Prognosis Relapsed Multiple Myeloma. J Clin Oncol 2018;36:2267-80.

2. Carpenter RO, Evbuomwan MO, Pittaluga S, et al. B-cell maturation antigen is a promising target for adoptive T-cell therapy of multiple myeloma. Clin Cancer Res 2013;19:2048-60.

3. Tai YT, Mayes PA, Acharya C, et al. Novel anti-Bcell maturation antigen antibody-drug conjugate (GSK2857916) selectively induces killing of multiple myeloma. Blood 2014;123:3128-38.

4. An G, Acharya C, Feng X, et al. Osteoclasts promote immune suppressive microenvironment in multiple myeloma: therapeutic implication. Blood 2016;128:1590-603.

5. Tai YT, Acharya C, An G, et al. APRIL and BCMA promote human multiple myeloma growth and immunosuppression in the bone marrow microenvironment. Blood 2016;127:3225-36.

6. Tai YT, Anderson KC. Targeting B-cell maturation antigen in multiple myeloma. Immunotherapy 2015;7:1187-99.

7. Cho SF, Anderson KC, Tai YT. Targeting B Cell Maturation Antigen (BCMA) in Multiple Myeloma: Potential Uses of BCMA-Based Immunotherapy. Front Immunol 2018;9:1821.

8. June CH, Sadelain M. Chimeric Antigen Receptor Therapy. N Engl J Med 2018;379:64-73.

9. June CH, O'Connor RS, Kawalekar OU, et al. CAR $\mathrm{T}$ cell immunotherapy for human cancer. Science 2018;359:1361-5.

10. Imai $\mathrm{C}$, Mihara K, Andreansky M, et al. Chimeric receptors with 4-1BB signaling capacity provoke potent cytotoxicity against acute lymphoblastic leukemia. Leukemia 2004;18:676-84.

11. Kochenderfer JN, Dudley ME, Feldman SA, et al. B-cell depletion and remissions of malignancy along with cytokine-associated toxicity in a clinical trial of anti-CD19 chimeric-antigen-receptor-transduced T cells. Blood 2012;119:2709-20.

12. Davila ML, Riviere I, Wang X, et al. Efficacy and toxicity management of 19-28z CAR T cell therapy in B cell acute lymphoblastic leukemia. Sci Transl Med 2014;6:224ra25.

13. Ali SA, Shi V, Maric I, et al. T cells expressing an anti-Bcell maturation antigen chimeric antigen receptor cause remissions of multiple myeloma. Blood 2016;128:1688-700.

14. Cohen AD, Garfall AL, Stadtmauer EA, et al. B-Cell Maturation Antigen (BCMA)-Specific Chimeric Antigen Receptor T Cells (CART-BCMA) for Multiple Myeloma (MM): Initial Safety and Efficacy from a Phase I Study. Blood 2016;128:1147.

15. Maus MV, June CH. Zoom Zoom: racing CARs for multiple myeloma. Clin Cancer Res 2013;19:1917-9.

16. Maus MV, June CH. Making Better Chimeric Antigen Receptors for Adoptive T-cell Therapy. Clin Cancer Res 2016;22:1875-84.

17. Lin L, Xing L, Acharya CM, et al. CD8+ Anti-BCMA mRNA CAR T-Cells Effectively Kill Human Multiple Myeloma Cells In Vitro and In Vivo. Blood 2017;130:3067.

18. Dolgin E. CAR-Ts move beyond B-cell cancers to myeloma. Nat Biotechnol 2017;35:599-601.

19. Yang Y, Jacoby E, Fry TJ. Challenges and opportunities of allogeneic donor-derived CAR T cells. Curr Opin Hematol 2015;22:509-15.

20. Berdeja JG, Lin Y, Raje N, et al. Durable Clinical Responses in Heavily Pretreated Patients with Relapsed/ Refractory Multiple Myeloma: Updated Results from a Multicenter Study of bb2121 Anti-Bcma CAR T Cell Therapy. Blood 2017;130:740.

21. James SE, Orgun NN, Tedder TF, et al. Antibodymediated B-cell depletion before adoptive immunotherapy with T cells expressing CD20-specific chimeric T-cell receptors facilitates eradication of leukemia in immunocompetent mice. Blood 2009;114:5454-63.

22. Wallen H, Thompson JA, Reilly JZ, et al. Fludarabine modulates immune response and extends in vivo survival of adoptively transferred CD8 T cells in patients with metastatic melanoma. PLoS One 2009;4:e4749.

23. Brentjens RJ, Riviere I, Park JH, et al. Safety and persistence of adoptively transferred autologous CD19- 
targeted $\mathrm{T}$ cells in patients with relapsed or chemotherapy refractory B-cell leukemias. Blood 2011;118:4817-28.

24. Zweegman S, Engelhardt M, Larocca A, et al. Elderly patients with multiple myeloma: towards a frailty approach? Curr Opin Oncol 2017;29:315-21.

Cite this article as: Cho SF, Anderson KC, Tai YT. BCMA CAR T-cell therapy arrives for multiple myeloma: a reality. Ann Transl Med 2018;6(Suppl 2):S93. doi: 10.21037/atm.2018.11.14
25. Fan F, Zhao W, Liu J, et al. Durable remissions with BCMA-specific chimeric antigen receptor (CAR)-modified $\mathrm{T}$ cells in patients with refractory/relapsed multiple myeloma. J Clin Oncol 2017;35:LBA3001. 